\title{
BMJ Global Health Healthcare use and expenditure for diabetes in Bangladesh
}

To cite: Shariful Islam SM Lechner A, Ferrari U, et al. Healthcare use and expenditure for diabetes in Bangladesh. BMJ Global Health 2017;2:e000033. doi:10.1136/bmjgh-2016000033

Received 4 February 2016 Revised 12 May 2016 Accepted 13 May 2016

\section{CrossMark}

For numbered affiliations see end of article.

\section{Correspondence to} Dr Sheikh Mohammed Shariful Islam; shariful.islam@icddrb.org

\section{ABSTRACT}

Background: Diabetes imposes a huge social and economic impact on nations. However, information on the costs of treating and managing diabetes in developing countries is limited. The aim of this study was to estimate healthcare use and expenditure for diabetes in Bangladesh.

Methods: We conducted a matched case-control study between January and July 2014 among 591 adults with diagnosed diabetes mellitus (DMs) and 591 age-matched, sex-matched and residence-matched persons without diabetes mellitus (non-DMs). We recruited DMs from consecutive patients and non-DMs from accompanying persons in the Bangladesh Institute of Health Science (BIHS) hospital in Dhaka, Bangladesh. We estimated the impact of diabetes on healthcare use and expenditure by calculating ratios and differences between DMs and non-DMs for all expenses related to healthcare use and tested for statistical difference using Student's t-tests.

Results: DMs had two times more days of inpatient treatment, 1.3 times more outpatient visits, and 9.7 times more medications than non-DMs (all $p<0.005$ ). The total annual per capita expenditure on medical care was 6.1 times higher for DMs than non-DMs (US\$635 vs US\$104, respectively). Among DMs, 9.8\% reported not taking any antidiabetic medications, $46.4 \%$ took metformin, $38.7 \%$ sulfonylurea, $40.8 \%$ insulin, $38.7 \%$ any antihypertensive medication, and $14.2 \%$ took antilipids over the preceding 3 months.

Conclusions: Diabetes significantly increases healthcare use and expenditure and is likely to impose a huge economic burden on the healthcare systems in Bangladesh. The study highlights the importance of prevention and optimum management of diabetes in Bangladesh and other developing countries, to gain a strong economic incentive through implementing multisectoral approach and cost-effective prevention strategies.

\section{INTRODUCTION}

In recent years, the burden of diabetes has increased worldwide with disproportionally high morbidity and mortality in developing countries. ${ }^{1}$ In Southeast Asia, current estimates indicate that $8.2 \%$ of the adult population, or 72.1 million people, have diabetes,

\section{Key questions}

What is already known about this topic?

- Diabetes is a costly condition.

- People with diabetes have higher healthcare use compared with matched persons withou diabetes.

- Diabetes has potential impacts on individuals and society.

What are the new findings?

- Healthcare expenditure in persons with diabetes in Bangladesh is six times higher than in persons without diabetes.

- Prevention and management of diabetes is likely to be a cost-saving approach for Bangladesh.

- Low use of statins is a concern.

Recommendations for policy

- More focus is needed for secondary prevention of complications.

- Proper management of diabetes and its risk factors is essential.

- There is a need to identify cost-effective strategies for prevention and management of diabetes.

which is projected to increase to 123 million by 2035 as a consequence of ongoing rapid urbanisation, lifestyle changes and increasing life expectancy. ${ }^{2}$ About half of the people with diabetes remain undiagnosed in this region, and a further 24.3 million people have pre-diabetes that will increase to 38.8 million by $2035 .^{2}$

Bangladesh has a total population of more than 160 million and is among the countries with the highest number of people with diabetes worldwide. ${ }^{3}$ The International Diabetes Federation (IDF) estimated 7.1 million people with diabetes in Bangladesh and almost an equal number with undetected diabetes. This number is estimated to double by 2025. ${ }^{3}$ In 2014, the Gross National Income purchasing power parity (PPP) in Bangladesh was US\$3330. The total government expenditure on health was $3.4 \%$ of 
total gross domestic product (GDP) in 2008 and per capita total expenditure on health was only US $\$ 15 .^{4}$ Diabetes care is mostly provided by the Diabetes Association of Bangladesh (DAB), which is a not-for-profit association of several hospitals and health centres across the country. The increasingly high incidence of diabetes is expected to have devastating social and economic impacts on the overburdened healthcare systems of the country.

Diabetes is a costly condition and can lead to several disabling and life-threatening complications, including stroke, heart attack, chronic kidney diseases, neuropathy, visual impairment and amputations. Studies in Bangladesh reported eye problems, chronic kidney diseases, cardiovascular diseases and depression as major complications. ${ }^{5-8}$ Although most of these complications can largely be prevented through the use of several inexpensive, easy-to-use and cost-effective interventions, their use in developing countries, where the majority of persons with diabetes live, remains tragically low. ${ }^{9}$ It is estimated that healthcare expenditure for diabetes accounts for $10.8 \%$ of the total annual healthcare expenditure worldwide, which totalled at least US $\$ 548$ billion in 2013 and is projected to exceed US $\$ 627$ billion by $2035 .^{2}$ The estimated healthcare cost of diabetes was on average US $\$ 5621$ per person in developed countries, compared with US\$356 in developing countries. ${ }^{2}$ A recent study by World Bank found $\$ 160$ per year in household expenses for diabetes care (2013 dollars) in Bangladesh.

Economic information on a disease can support the process of planning and resource allocation allowing cost-effective and efficient health spending. The social and economic impact of diabetes is complex and difficult to measure. Different methods and economic models have been used to measure the economic burden of chronic diseases, such as diabetes, but debates about the most appropriate methods are still ongoing. ${ }^{10}$ However, information on the healthcare use, costs, and social and economic impact of diabetes in developing countries is generally not available. In this context, we aimed to conduct for the first time a comprehensive matched case-control study on the economic impact of diabetes in Bangladesh. The primary objective was to estimate the total annual per-person expenditure for medical care among persons with and without diabetes in Bangladesh.

\section{MATERIALS AND METHODS \\ Study population and location}

We conducted a matched, case-control study including 591 persons with diagnosed diabetes mellitus (DMs) and 591 age-matched, sex-matched and residence-matched controls without diabetes mellitus (non-DMs) at the outpatient department (OPD) of the Bangladesh Institute of Health Science (BIHS) hospital between January and July 2014. A detailed methodology of the study design has been published elsewhere. ${ }^{11}{ }^{12}$ In short, BIHS is a 500-bed multidisciplinary hospital with one of the largest diabetes OPD visits by patients in a year in Bangladesh. BIHS is recognised as a national level tertiary healthcare and research institution for diabetes, and is affiliated with the DAB, which has a network of diabetes centres throughout the country. The BIHS hospital serves a population of about 2.2 million in Dhaka city and nearby districts. ${ }^{13}$

\section{Sample size and selection}

We considered a sample size of 500 cases and 500 controls to provide $90 \%$ power to detect a $5 \%$ difference in rates and proportions between cases and controls. Inclusion criteria for cases were: adults diagnosed with diabetes at BIHS OPD according to the WHO criteria, provision of anthropometric measurements, and written informed consent. Controls were individuals without a self-reported history of diabetes matched on a 1:1 basis to cases by area of residence, age (within a 5 -year band) and sex (male or female). We included all consecutive patients meeting the inclusion criteria waiting for consultation at the BIHS OPD. Controls were recruited within 48 hours of recruiting the index case, from either visitors of patients attending the OPD, or non-blood related visitors of index diabetes cases, in the same hospitals or the same geographical residence of cases. All controls underwent identical study questioning and examination as cases. One completed control interview was obtained for each case interview.

\section{Data collection}

Data were collected by a team consisting of a study physician, a research officer and three research assistants experienced in hospital data collection. The team was trained for 4 weeks on diabetes epidemiology, study protocol, interview skills, research ethics, physical measurements and blood pressure (BP) measurements. Data were collected through face-to-face interviews using structured questionnaires and by review of patients' medical records. The research tools and instruments used in this study were developed by the IDF Health Economic Group and translated into Bengali according to the WHO process of translation, back-translation and adaptation of research instruments. ${ }^{14}$ The questionnaires were field tested in a similar setting at the OPD of Bangladesh Institute of Research on Diabetes, Endocrine and Metabolism (BIRDEM) hospital before conducting the interviews among 25 cases and 25 control subjects. Feedback from the field tests was used to improve the language and the contents of the questionnaire and tools, as well as to adapt them to local circumstances based on previous validated survey items. ${ }^{4}$

The interview included questions about: socioeconomic status, quality of life, self-satisfaction with health, self-reported diseases, healthcare use, direct and indirect costs, tobacco use, impact of health problems, sources of funds, mental health, physical measurements, BP and medication use. Physical measurements of 
weight, height, waist circumference and hip circumference were conducted according to standard medical protocols. BP was measured using a digital BP monitor (Omron, SEM-1, Omron Corporation, Japan). Two repeated measurements were recorded within an interval of $5 \mathrm{~min}$, alternating the right and left arms. The average of the two readings was considered. Hypertension was defined as systolic $\mathrm{BP}>140 \mathrm{~mm} \mathrm{Hg}$ and/or diastolic $\mathrm{BP}>90 \mathrm{~mm} \mathrm{Hg}$.

The estimation of expenditures for medical care relied on participant recall to ascertain charges for medicines, supplies and use of any medical care services. To increase accuracy of recall, we asked about events occurring only during the previous 90 days. We further attempted to improve the temporal accuracy by asking respondents to name and associate a well-remembered event that had occurred $\sim 90$ days prior to the interview date. To estimate expenditures for medicines, we asked participants to show us all medicines they were currently taking and the most recent prescription and medical records they received from their doctors, so that we could record all prescription medicines taken by the participant. We then calculated the unit price of each medicine using the Bangladesh online medicine price index database and costs for medicines per day for each participant and multiplied by 365 to get the annual costs for medicines. For overnight admissions to hospital (inpatient visits) and visits to OPDs, respondents were asked to recall the amount of total payment, including payment for medicines and tests or only a portion of the total bill or charge. This subset of information from a single visit was used to estimate the characteristics of all events of the same kind, including mean length of inpatient admission and mean payments per admission, per OPD visits and per purchase of medicine in case of inpatient visits. The total annual point-of-care service payment per capita was calculated as the sum of total annual payment per patient for inpatient care, OPD care, testing for glucose strips and medicines.

\section{Ethics}

The objectives and importance of the research were explained to all participants before recruitment. Participation was voluntary and written informed consent was obtained from all participants. All data sets containing information on the participants were locked and the identity of individual participants was kept confidential. The study was approved by the Research Review Committee and Ethical Review Committee of the International Center for Diarrheal Diseases Research, Bangladesh (PR-13062) and obtained ethical clearance waiver from Ludwig-Maximilians-Universität (LMU) and BIHS.

\section{Data entry, verification and analysis}

All data forms and questionnaires were checked for errors and necessary corrections were made before data entry. Data were entered electronically using Microsoft Access followed by a wide range of consistency checks.
The reliability of the data entry program was verified by randomly comparing the data with hard-copy records by study investigators. Prior to analysis, data were checked by a statistician for range, consistency and normality. Suspected values were all rechecked against the hardcopy records by the study team and excluded from the analysis data set if a correct response could not be found. Prior to substantive data analysis, we reduced two extreme outlier values to the level of the next largest original value. We checked the hospital data and considered the extreme values to be most probably erroneous and therefore unreliable. Also, prior to analysis, to prevent bias arising from rare and uncharacteristically long hospital stays, we winsorized the hospitalisation data by reducing two extreme outlier values to the level of the next largest unmodified value in the data set which has previously been used in similar studies. ${ }^{15} 16$ Descriptive analysis was performed for all variables and unadjusted comparisons between cases and controls were performed using Student's t-tests (for continuous variables) or $\chi^{2}$ tests (for discrete variables). We estimated the impact of diabetes on healthcare use by calculating ratios and differences between DMs and non-DMs and tested for statistical difference using Student's t-tests. All statistical analyses were performed using SPSS V.20 (IBM Corporation, USA).

\section{RESULTS}

A total of 1265 participants were approached, and 1240 (98\%) agreed to participate. Altogether, 40 patients were excluded-including 15 controls who had a history of diabetes-8 patients were pregnant and 17 patients had no medical records available at the time of data collection. Data were collected from 1200 participants. During analysis, 18 participants were excluded due to matching problems and incomplete information for a total of 1182 participants included in the final analysis (figure 1). The overall mean $\pm \mathrm{SD}$ age of the 1182 participants was $50.4 \pm 11.4$ years (table 1 ). DMs were, on average, 2 years older than non-DMs $(\mathrm{p}=0.004)$, less likely to be married $(80.5 \%$ vs $87.5 \%, \mathrm{p}=0.001)$, less likely to have completed higher education (year 12 and above) $(30.8 \%$ vs $40.8 \%, \mathrm{p}=0.001)$ and less likely to be engaged in an occupation as service or business $(28.8 \%$ vs $42.0 \%, \mathrm{p}=0.001)$. Although the cases and controls were not directly matched on family income, we observed similar self-reported annual family incomes per capita, averaging US $\$ 1256.4 \pm 1307.7$ for DMs and US $\$ 1326.9 \pm 1971.8$ for non-DMs (US\$1=78 Bangladesh Taka (BDT), 2015 Bangladesh Bank).

\section{Use of inpatient care}

As shown in table 2, $14.2 \%$ of DMs reported at least one hospital inpatient admission during the past year, compared with $6.6 \%$ of non-DMs, which was statistically significant $(\mathrm{p}=<0.001)$ with a ratio of 2.2. The mean number of admissions during the past 1 year among participants 


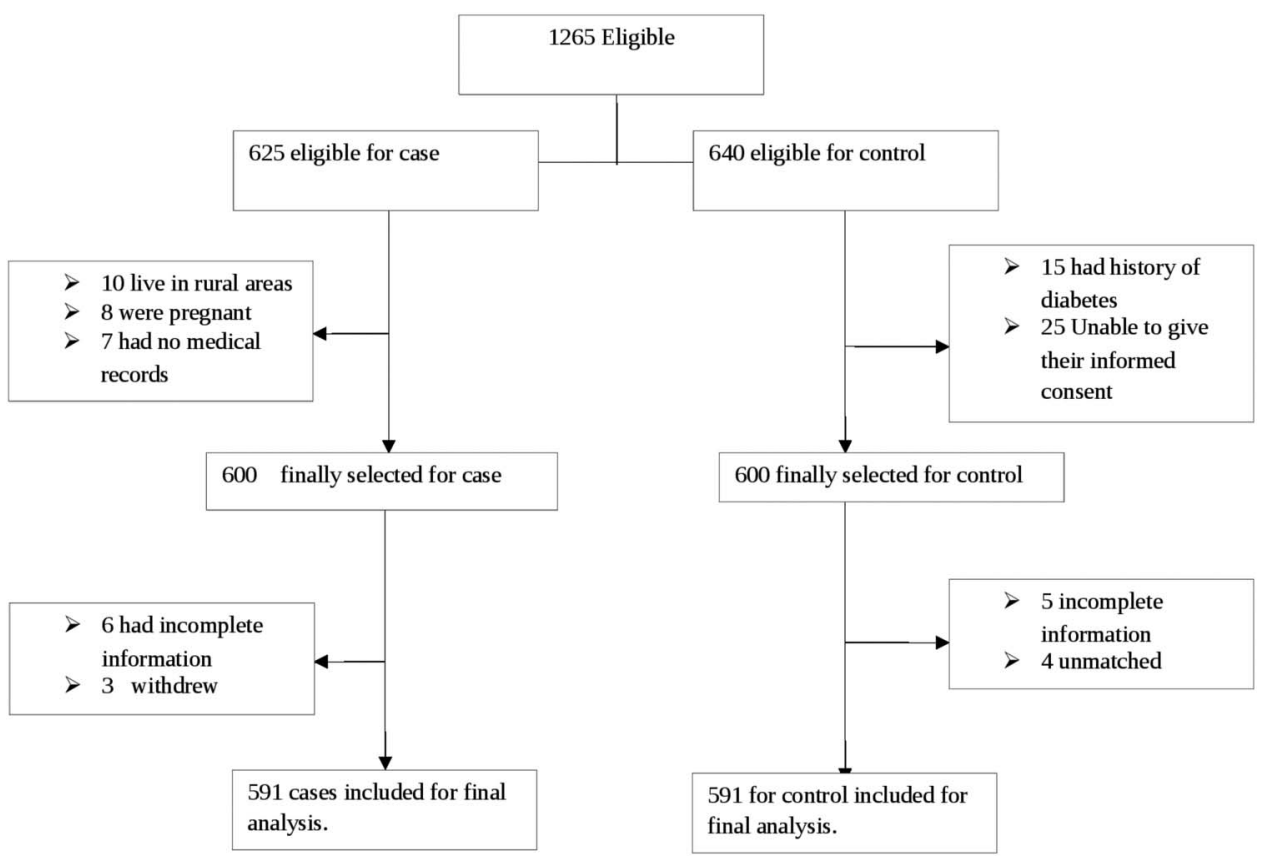

Figure 1 Flow chart.

admitted was 1.4 days for DMs and 1.6 days for non-DMs, which was statistically not significant $(\mathrm{p}=0.388)$.

The mean length of stay for the most recent hospital admission among participants admitted was almost the same length for DMs and non-DMs (4.9 vs 4.8 days, $\mathrm{p}=0.878$ ). For all DMs in the sample, the mean annual inpatient days were 0.9 days, which was calculated as the product of mean length of stay and estimated annual admissions per person with diabetes, divided by the total number of DMs in the sample. For non-DMs, the corresponding figure was 0.5 days per person per year. The ratios for annual inpatient days per person, for DMs and non-DMs, were 2.0. The mean annual inpatient admissions per person for all participants were double for DMs than non-DMs ( 0.2 vs 0.1 days $)$ and statistically significant $(p=0.008)$.

\section{Use of outpatient care}

Table 2 shows the self-reported use of outpatient services by the study participants. DMs had more than four times the mean annual visits to OPD compared with non-DMs. Almost all OPD visits by DMs were to hospital-based OPD clinics: 4.1 of the 6.2 visits. For non-DMs, the most visits were to specialists/surgeons: 1.0 of the 4.6 visits. Both DMs and non-DMs reported $<1 \%$ mean annual visits to MBBS doctors, traditional healers, pharmacy counters, and community health workers.

\section{Use of medicines}

Table 2 describes the use of medicines by DMs and non-DMs. Almost all $(97.8 \%)$ DMs reported taking at least one medicine at the time they were interviewed, compared with one-fifth (20.6\%) of non-DMs, which was highly statistically significant $(p<0.001)$. The mean number of medicines among persons taking any medicine was also significantly different between DMs (3.7 medicines $)$ and non-DMs $(1.8$ medicines, $\mathrm{p}<0.001)$. Use of medicines increased with age and with the length of time since diagnosis of DM (both $\mathrm{p}<0.001$ ). The mean number of medicines taken per person among all participants was also statistically significant among DMs and non-DMs $(p<0.001)$ and increased steadily with age and duration of diabetes.

\section{Payment for services}

As shown in table 3, DMs reported paying 6.1 times as much for total annual medical care services during the preceding year as non-DMs (US\$635 vs US\$104). For DMs, payments were much higher for inpatient services than outpatient services compared with non-DMs. However, DMs paid 2.1 times more than non-DMs for hospital services, in part because payments per admission were about 1.5 times as high for DMs as for non-DMs. In the case of payments for OPDs, the ratio of payments for DMs to payments by non-DMs was less than for inpatient care but still substantial $(2.1,1.5)$. Annual total payments for medicines was 35385 BDT (US\$454) per person for DMs and 1609 BDT (US\$21) for non-DMs. DMs ended up paying almost 22 times more annually for medicines than non-DMs, which was highly statistically significant $(p<0.001)$. Total annual payment per person for medicines was the largest cost for DMs, and for non-DMs it was the annual payment for OPDs.

\section{Use of essential medicines}

Table 4 summarises the current use of medicines by DMs by age range and length of time since diagnosis. Among DMs, 9.8\% reported not taking any anti-diabetic 
Table 1 Characteristics of study participants

\begin{tabular}{|c|c|c|c|c|}
\hline Variables & DMs n (\%) & Non-DMs n (\%) & Total n (\%) & p Value \\
\hline \multicolumn{5}{|l|}{ Age, years } \\
\hline$($ Mean $\pm S D)$ & $51.4 \pm 11.6$ & $49.5 \pm 11.1$ & $50.4 \pm 11.4$ & 0.004 \\
\hline $40-49$ & $144(24.4)$ & $145(24.5)$ & $289(24.5)$ & \\
\hline $50-59$ & $193(32.7)$ & 214 (36.2) & 407 (34.4) & \\
\hline \multicolumn{5}{|l|}{ Sex } \\
\hline Male & $255(43.1)$ & $255(43.1)$ & $510(43.1)$ & 1.000 \\
\hline Female & $336(56.9)$ & $336(56.9)$ & $672(56.9)$ & \\
\hline \multicolumn{5}{|l|}{ Marital status } \\
\hline Married & $476(80.5)$ & $517(87.5)$ & $993(84.0)$ & 0.001 \\
\hline Primary & $103(17.4)$ & $96(16.2)$ & $199(16.8)$ & \\
\hline Secondary & $190(32.1)$ & $178(30.1)$ & $368(31.1)$ & \\
\hline Higher secondary and above & $182(30.8)$ & $241(40.8)$ & $423(35.8)$ & \\
\hline \multicolumn{5}{|l|}{ Occupation } \\
\hline Unemployed & $6(1.0)$ & $9(1.5)$ & $15(1.3)$ & 0.001 \\
\hline Service or business & $170(28.8)$ & $248(42.0)$ & $418(35.4)$ & \\
\hline Housewife & $309(52.3)$ & 271 (45.9) & $580(49.1)$ & \\
\hline Others & $106(17.9)$ & $63(10.7)$ & $169(14.3)$ & \\
\hline \multicolumn{5}{|l|}{ Family size } \\
\hline$>30000 \mathrm{BDT}$ & $193(36.3)$ & $200(36.2)$ & $393(36.3)$ & \\
\hline \multicolumn{5}{|l|}{ Diabetes duration, years } \\
\hline Median (Q1, Q3) & $6(3,11)$ & NA & $6(3,11)$ & NA \\
\hline$<5$ & $248(42.0)$ & NA & $248(42.0)$ & NA \\
\hline $5-10$ & $194(32.8)$ & NA & $194(32.8)$ & \\
\hline$>10$ & $149(25.2)$ & NA & 149 (25.2) & \\
\hline
\end{tabular}

DMs, patients with diagnosed diabetes mellitus; NA, not applicable; Non-DMs, patients without diabetes mellitus; Q1, quartile 1.

medicine at the time of interview. The most commonly used anti-diabetic medicine was metformin $(46.9 \%)$, followed by insulin $(40.8 \%)$ and sulfonylurea $(38.7 \%)$. Only $38.7 \% \%$ of participants with diabetes used any anti-hypertensive medication. $\beta$-Blockers were the most common $(25.9 \%)$ anti-hypertensives used. Only $14.2 \%$ of participants with diabetes used any statins, and $7.6 \%$ reported use of any anticoagulants. Almost one-fifth $(20.5 \%)$ of DMs were taking vitamins and more than half $(57 \%)$ used other medications.

\section{DISCUSSION}

This study highlights the large economic burden of diabetes on individuals and healthcare systems in
Bangladesh. To the best of our knowledge, this is the first-ever published matched case-control study of healthcare use and expenditure for diabetes in South Asia. Our results show that the use of healthcare services and medicines was dramatically higher among DMs than among matched non-DMs. DMs reported twice as many inpatient admissions and annual inpatient treatment days, 1.3 times more annual outpatient visits and 9.7 times more prescription medicines compared with non-DMs. Using the IDF estimates of 8.4 million DMs in Bangladesh, the total estimated healthcare expenditure for diabetes in Bangladesh is around US $\$ 5.3$ billion. We estimated that healthcare expenditure, based on total annual point-of-service payments, was 6.12 times higher 
Table 2 Use of inpatient and outpatient services, and medicines by the study participants

\begin{tabular}{|c|c|c|c|c|c|}
\hline & DMs & Non-DMs & Ratio & $\begin{array}{l}\text { Mean difference } \\
(95 \% \mathrm{Cl})\end{array}$ & p Values \\
\hline \multicolumn{6}{|l|}{ Use of inpatient services } \\
\hline $\begin{array}{l}\text { Mean number of admissions, last } 1 \text { year, among participants } \\
\text { admitted }\end{array}$ & 1.4 & 1.6 & 0.9 & $-0.2(-0.8$ to 0.3$)$ & 0.388 \\
\hline Mean annual inpatient days per person, all participants & 0.9 & 0.5 & 2.0 & $0.5(-0.0$ to 0.9$)$ & 0.053 \\
\hline Mean annual inpatient admissions per person, all participants & 0.2 & 0.1 & 2.0 & 0.1 (0.0 to 0.2$)$ & 0.008 \\
\hline \multicolumn{6}{|l|}{ Use of outpatient services } \\
\hline $\begin{array}{l}\text { Estimated percentage of participants with } \geq 1 \text { outpatient visit } \\
\text { (last } 90 \text { days) }\end{array}$ & 59.2 & 13.7 & 4.3 & 45.5 (35.5 to 55.5) & 0.271 \\
\hline $\begin{array}{l}\text { Mean annual visits to hospital outpatient departments, per } \\
\text { person, all participants }\end{array}$ & 4.1 & 0.8 & 4.9 & 3.3 (2.8 to 3.7$)$ & $<0.001$ \\
\hline $\begin{array}{l}\text { Mean annual visits to a specialist/surgeon, per person, all } \\
\text { participants }\end{array}$ & 0.7 & 1.0 & 0.7 & $-0.3(-0.7$ to -0.0$)$ & 0.040 \\
\hline $\begin{array}{l}\text { Mean annual visits to an MBBS doctor, per person, all } \\
\text { participants }\end{array}$ & 0.2 & 0.6 & 0.4 & $-0.4(-0.6$ to -0.2$)$ & 0.001 \\
\hline $\begin{array}{l}\text { Mean annual visits to a traditional healer/quack provider, per } \\
\text { person, all participants }\end{array}$ & 0.6 & 0.8 & 0.7 & $-0.2(-0.6$ to 0.2$)$ & 0.253 \\
\hline $\begin{array}{l}\text { Mean annual visits to a pharmacy counter, per person, all } \\
\text { participants }\end{array}$ & 0.6 & 0.8 & 0.7 & $-0.2(-0.6$ to 0.2$)$ & 0.273 \\
\hline Mean number of medicines taken per person, all participants & 3.6 & 0.4 & 9.7 & $3.2(3.1$ to 3.4$)$ & $<0.001$ \\
\hline
\end{tabular}

DMs, patients with diagnosed diabetes mellitus; Non-DMs, patients without diabetes mellitus.

Table 3 Payments for medical care (2014 BDT)

\begin{tabular}{|c|c|c|c|c|c|}
\hline Payments for medical care (2014 BDT) & DMs & Non-DMs & Ratio & Mean difference $(95 \% \mathrm{Cl})$ & p Values \\
\hline Mean payment per inpatient admission, if admitted & 35672 & 24735 & 1.4 & $10937(-1914$ to 23787$)$ & 0.106 \\
\hline Total annual payment per person for inpatient care & 6592 & 3088 & 2.1 & $3504(-3882$ to 10890$)$ & 0.339 \\
\hline Total annual payment per person for outpatient care & 5075 & 3397 & 1.5 & $1678(-1869$ to 5226$)$ & 0.352 \\
\hline Total annual payment per person for medicines & 35385 & 1609 & 22.0 & 33776 (29 977 to 37575$)$ & $<0.001$ \\
\hline Total annual point-of-service payments/charges & 49538 & 8094 & 6.1 & 41443 (26 710 to 56176$)$ & $<0.001$ \\
\hline
\end{tabular}

DMs, patients with diagnosed diabetes mellitus; NA, not applicable; Non-DMs, patients without diabetes mellitus.

among DMs than among non-DMs. The impact of the diabetes study in China, which used similar methods to our study, showed that point-of-service payments were 4.0 times higher among DMs than among non-DMs. ${ }^{9} \mathrm{~A}$ study in India reported an annual mean direct cost for diabetes of US $\$ 380$, which was less than that reported by our study. ${ }^{17}$ The results of our study show a much higher expenditure ratio in Bangladesh compared with China and other developed countries where the ratio of expenditure for diabetes ranged from 2.0 to $2.5 .^{18-20}$ 
Table 4 Current use of medicines by participants with diabetes

\begin{tabular}{|c|c|c|c|c|c|c|c|c|c|c|}
\hline \multirow[t]{2}{*}{ Medicine category } & \multicolumn{4}{|c|}{ Age category (years) } & \multirow[t]{2}{*}{ p Value } & \multicolumn{3}{|c|}{ DM duration (years) } & \multirow[t]{2}{*}{ Total } & \multirow[t]{2}{*}{ p Value } \\
\hline & $<40$ & $40-49$ & $50-59$ & $\geq 60$ & & $<5$ & $5-10$ & $>10$ & & \\
\hline Anti-diabetes & 87.5 & 91.7 & 87.0 & 94.3 & 0.097 & 87.5 & 87.1 & 98.7 & 90.2 & $<0.001$ \\
\hline Metformin & 56.2 & 52.1 & 46.1 & 37.3 & 0.013 & 49.2 & 46.9 & 43.0 & 46.9 & 0.483 \\
\hline Sulfonylurea & 37.5 & 35.4 & 40.9 & 39.9 & 0.751 & 39.5 & 37.1 & 39.6 & 38.7 & 0.850 \\
\hline Insulin & 37.5 & 42.4 & 38.9 & 43.7 & 0.701 & 29.8 & 40.7 & 59.1 & 40.8 & 0.000 \\
\hline Other anti-diabetes & 0.0 & 4.2 & 3.6 & 5.7 & 0.088 & 2.8 & 3.6 & 5.4 & 3.7 & 0.429 \\
\hline Anti-hypertensives & 13.5 & 33.3 & 46.1 & 50.0 & 0.000 & 29.0 & 43.3 & 49.0 & 38.7 & $<0.001$ \\
\hline$\beta$-blockers & 9.4 & 20.8 & 31.1 & 34.2 & 0.000 & 18.1 & 29.9 & 33.6 & 25.9 & 0.001 \\
\hline Diuretics & 0.0 & 0.0 & 2.1 & 1.3 & 0.257 & 0.8 & 1.0 & 1.3 & 1.0 & 0.879 \\
\hline ARB & 2.1 & 4.9 & 7.3 & 9.5 & 0.094 & 4.4 & 8.2 & 7.4 & 6.4 & 0.231 \\
\hline CCB & 0.0 & 2.1 & 1.0 & 1.3 & 0.594 & 1.6 & 1.0 & 0.7 & 1.2 & 0.799 \\
\hline ACE inhibitors & 1.0 & 5.6 & 3.1 & 3.2 & 0.324 & 3.2 & 4.6 & 2.0 & 3.4 & 0.401 \\
\hline Other anti-hypertensives & 1.0 & 7.6 & 7.3 & 6.3 & 0.091 & 6.0 & 4.6 & 8.1 & 6.1 & 0.423 \\
\hline Anti-lipids (statins) & 3.1 & 11.8 & 17.1 & 19.6 & 0.000 & 10.5 & 17.5 & 16.1 & 14.2 & 0.082 \\
\hline Anti-coagulants & 2.1 & 5.6 & 9.3 & 10.8 & 0.034 & 4.4 & 9.3 & 10.7 & 7.6 & 0.041 \\
\hline GIT medicines & 7.3 & 16.7 & 13.0 & 13.9 & 0.211 & 16.1 & 13.4 & 8.1 & 13.2 & 0.070 \\
\hline Antibiotics & 1.0 & 0.0 & 1.0 & 1.9 & 0.395 & 0.8 & 0.5 & 2.0 & 1.0 & 0.447 \\
\hline Anti-thyroids & 2.1 & 3.5 & 3.6 & 6.3 & 0.416 & 3.2 & 4.6 & 4.7 & 4.1 & 0.682 \\
\hline Vitamins & 19.8 & 20.1 & 17.1 & 25.3 & 0.300 & 18.5 & 19.1 & 25.5 & 20.5 & 0.211 \\
\hline Other medicines & 43.8 & 66.7 & 52.8 & 61.4 & 0.002 & 58.9 & 50.0 & 63.1 & 57.0 & 0.039 \\
\hline
\end{tabular}

ARB, angiotensin receptor blocker; CCB, calcium channel blocker; DM, diabetes mellitus; GIT, gastro intestinal tract.

Among South Asians, diabetes and its complications start relatively early with a higher magnitude and severity compared with Western populations. ${ }^{21} 22$ A recent study in Bangladesh showed that relatively newly diagnosed patients with type 2 diabetes presenting at the OPD of a hospital had several complications. ${ }^{5}$ As a result, patients with diabetes in the clinic are much sicker than their counterparts in other places and require more medications and healthcare services. In addition, a greater number of patients with diabetes presenting at the clinics in Bangladesh had uncontrolled diabetes, poor knowledge and were underusing antihypertensives and statins. $^{5} 23$ All these factors might have resulted in expensive, disabling complications and higher use of medical service as found in our study.

In Bangladesh, as in most developing countries, barriers to public health facilities force the poor to pay for healthcare out of pocket, often driving them further into poverty. ${ }^{24}$ As a result, DMs may not seek the required preventive care, which further increases the risk of complications and is more costly (if treated at all). Our participants with diabetes did not use more inpatient or outpatient services nor take more medicines than people with diabetes in China. ${ }^{26}{ }^{27}$ However, the controls used very few medicines and services relative to non-DMs in other countries. ${ }^{15}$ This underuse of medical care by the general population is a third driver of the large diabetes-associated differences that we report. Medical care in Bangladesh is very costly relative to an average person's mean family income, often difficult to access, and leaves a household vulnerable to the effects of catastrophic health expenses. ${ }^{24} 2528$ Bangladesh has an opportunity to reduce future medical care costs by diagnosing diabetes earlier and by using inexpensive generic medicines much more widely and thus reducing hospitalisations, disability and mortality. ${ }^{29}{ }^{30}$ Our data suggest that DMs in Bangladesh are less likely to receive preventive services and medication for proper management of diabetes and its complications, and therefore their high use of inpatient services might be the unfortunate result.

Our study has several limitations that should be kept in mind when using and interpreting its results. This is an observational cross-sectional study that estimates the expenditures and other effects caused by diabetes from a case-control rather than an experimental comparison. Data were collected from the consecutive patients with diabetes and matched controls at the OPD of one large hospital in Dhaka where people from mostly nearby regions come for treatment, and thus the results do not represent Bangladesh as a whole. This type of sampling might include some bias. However, this hospital is affiliated with the $\mathrm{DAB}$ and healthcare costs are similar to all DAB affiliated healthcare centres in Bangladesh. We excluded undiagnosed cases of diabetes and prediabetes; therefore, the differences and ratios reported here are probably an overestimate of medical services usage per person among persons with undiagnosed diabetes and pre-diabetes. It is likely that DMs in our samples were mostly from urban areas, had more 
complications and were more likely to use and to be able to afford healthcare services than the general diabetes population. A major limitation of this study is recall bias by cases and controls about different costs associated with disease and hospitalisation. Some participants might have failed to differentiate costs for treatment of each individual item mentioned in the questionnaire, which might affect the results of the study. In addition, although the case and controls differed substantially in respect of marital status, education and occupation, these differences are not relevant to the analysis and unlikely to change the results presented.

In this study, we minimised under-reporting by limiting counts of both visits and hospitalisations to a 90-day window and asking about the details of visits and hospitalisations for only the most recent encounter within that period. Since DMs had much more encounters than controls, any under-reporting in our data should have the effect of downwardly biasing our estimates of the effects of diabetes on medical care use. We also performed hospital audits and collected information from multiple sources to verify the correctness of information provided, to the extent possible. Regarding medication use, we asked participants to show us the medicines they were currently taking and matched with the current prescription. Another limitation is that the presented estimation of the burden does not include the intangible costs of diabetes, such as pain experienced, human suffering and the reduced quality of life, as well as some of the non-medical costs attributed to diabetes such as travel costs, time spent for travel and consultation, special diets and costs associated with informal caregivers. A unique strength of this study is its matched case-control design, which reduced the confounding bias of age, sex and residence during the recruitment stage and is the most widely used and accepted approach for measuring the economic and social impacts of diabetes. ${ }^{19} 20$ Despite our matching efforts, there were some differences between cases and controls, such as age matching was not perfectly balanced and this could have introduced bias. However, residence and sex of the participants were very similar across our cases and controls, as would be expected, and both groups were also similar on family income per person and educational attainment.

The results of our study also suggest, in line with previous studies from China, that the social and economic impacts of diabetes might be much higher in developing countries compared with developed countries, where diabetes is diagnosed at an early stage and treatment reaches the target population more effectively. ${ }^{9}$ Therefore, prevention strategies using low-cost, innovative information technology might be a possible approach to improve the health systems for diabetes. ${ }^{31-33}$ The IDF estimated the healthcare costs attributed by diabetes in developing countries to be US\$356 in 2013, based on an assumed age-sex adjusted diabetes to nondiabetes ratio of 2.0 per year per capita. ${ }^{2}$ If the adjusted expenditure ratio of 6.1 that we observed had instead been used, the IDF estimate would have been much higher. The expenditure ratios reported here imply, as do analyses using different methods, that in developing countries the economic burden of diabetes may constrain the availability of medical resources for other health conditions and impede national economic growth in future years. In Bangladesh, the potential health and economic impact of diabetes will be particularly large, because the number of DMs in Bangladesh is growing rapidly and because of lifestyle changes as a result of rural to urban migration. ${ }^{34}$

\section{CONCLUSION}

The results of our study indicate that diabetes is likely to increase healthcare use and expenditure in Bangladesh. However, larger studies across the country are needed to better understand its social and economic burden. The overall healthcare expenditure and health system impact of diabetes in Bangladesh found by this study is much higher than previous estimates by international organisations. The study highlights the importance of prevention and optimum management of diabetes in Bangladesh, and other developing countries, in order to gain a strong economic incentive through implementing a multisectoral approach and cost-effective prevention strategies.

\section{Author affiliations}

${ }^{1}$ NCD Unit, International Center for Diarrhoeal Diseases Research, Bangladesh (ICDDR,B), Dhaka, Bangladesh

${ }^{2}$ Center for International Health $(\mathrm{ClH})$, Ludwig-Maximilians-Universität (LMU), Munich, Germany

${ }^{3}$ Cardiovascular Division, The George Institute for Global Health, Sydney Medical School, University of Sydney, Sydney, Australia

${ }^{4}$ Diabetes Center, Diabetes Research Group, Medizinische Klinik und Poliklinik IV, Ludwig-Maximilians-Universität, Munich, Germany

${ }^{5}$ Institute of Health Economics and Health Care Management, Helmholtz Zentrum München $(\mathrm{GmbH})$, German Research Center for Environmental Health, Munich, Germany

${ }^{6}$ German Center for Diabetes Research (DZD), Germany

${ }^{7}$ Kaiser Permanente Center for Health Research, Portland, Oregon, USA

${ }^{8}$ Centre for Applied Health Research and Delivery, Liverpool School of Tropical Medicine, Liverpool, UK

\section{Handling editor Sanni Yaya}

Acknowledgements The authors thank all the participants for their time and the field staff for assisting in data collection. They express their deep thanks and gratitude to Professor MHA Rouf, Director, BIHS Hospital and colleagues from BIHS for support in data collection. They thank Professor Fabio Zicker, Senior Visiting Professor, International Health, Center for Technological Development in Health (CDTS) at Fiocruz Foundation, Rio de Janeiro, Brazil and Ms Natalie Linton, Oregon State University, USA for editorial assistance. This paper has appeared in an earlier form as a dissertation available at https://edoc.ub.uni-muenchen.de/19148/1/Shariful_Islam_Sheikh_ Mohammed.pdf. ${ }^{12}$

Contributors SMSI, the principal investigator, was involved in the concept, design, development of the study and the instruments, as well as in the implementation, analysis and reporting aspects of the study. RH, LWN and $A L$ were involved in all aspects of the study and provided expert advice for the study design and writing of the manuscript. UF and JS were involved in the 
study design and review of the protocol. ML revised the protocol, assisted in data analysis and provided suggestions to improve the study design and manuscript. All authors have read and approved the final version of the manuscript.

Funding This research study was funded by ICDDR,B's core SIDA grant number GR-01014. ICDDR,B also gratefully acknowledges the following donors who provided unrestricted support: Australian Agency for International Development (AusAID), Government of the People's Republic of Bangladesh, Canadian International Development Agency (CIDA), Swedish International Development Agency (SIDA), and the Department for International Development, UK (DFID). SMSI also received support from the Center for International Health $(\mathrm{ClH})$, Ludwig-Maximilians University of Munich, DAAD, $\mathrm{BMZ}$ and Exceed as a PhD scholar for this study.

Disclaimer The funding organisations had no role in the design and conduct of the study; collection, management, analysis and interpretation of data; preparation, review or approval of the manuscript; nor the decision to submit for publication.

Competing interests SMSI (MBBS, MPH, PhD) is a Senior Research Investigator at the Non-Communicable Diseases Unit, ICDDR, B and a Postdoc Research Fellow at the George Institute for Global Health, University of Sydney, Australia. He was a PhD Fellow at the Center for International Health, Ludwig-Maximilians-Universität (LMU), Munich, Germany. His research interests focus on developing low-cost sustainable solutions for diabetes and other non-communicable diseases. His research interests include large scale interventions study, clinical epidemiology, clinical trials, economic study and translational research on diabetes and cardiovascular diseases.

Patient consent Obtained.

Ethics approval ICDDR,B.

Provenance and peer review Not commissioned; externally peer reviewed.

Data sharing statement No additional data are available.

Open Access This is an Open Access article distributed in accordance with the Creative Commons Attribution Non Commercial (CC BY-NC 4.0) license, which permits others to distribute, remix, adapt, build upon this work noncommercially, and license their derivative works on different terms, provided the original work is properly cited and the use is non-commercial. See: http:// creativecommons.org/licenses/by-nc/4.0/

\section{REFERENCES}

1. Islam SMS, Purnat TD, Phuong NTA, et al. Non-communicable diseases (NCDs) in developing countries: a symposium report. Global Health 2014;10:81.

2. International Diabetes Federation. IDF DIABETES ATLAS. International Diabetes Federation. Brussels, Belgium: International Diabetes Federation, 2013:160.

3. International Diabetes Federation. IDF DIABETES ATLAS. International Diabetes Federation. Brussels, Belgium: International Diabetes Federation, 2011:144.

4. Dhillon PK, Jeemon P, Arora NK, et al. Status of epidemiology in the WHO South-East Asia region: burden of disease, determinants of health and epidemiological research, workforce and training capacity. Int J Epidemiol 2012;41:847-60.

5. Islam SMS, Alam DS, Wahiduzzaman M, et al. Clinical characteristics and complications of patients with type 2 diabetes attending an urban hospital in Bangladesh. Diabetes Metab Syndr 2015;9:7-13.

6. Islam SMS, Rawal LB, Niessen LW. Prevalence of depression and its associated factors in patients with type 2 diabetes: a cross-sectional study in Dhaka, Bangladesh. Asian J Psychiatr 2015;17:36-41.

7. Islam SMS, Ferrari U, Seissler J, et al. Association between depression and diabetes amongst adults in Bangladesh: a hospital based case-control study. J Glob Health 2015;5:020406.

8. Islam S, Islam M, Rawal L, et al. Clinical profile of patients with diabetic nephropathy in a tertiary level hospital in Dhaka, Bangladesh. Archieves Med Health Sci 2015;3:191-8.

9. Yang W, Zhao W, Xiao J, et al. Medical care and payment for diabetes in China: enormous threat and great opportunity. PLOS ONE 2012;7:e39513.
10. Zhang $\mathrm{P}$, Zhang $\mathrm{X}$, Brown J, et al. Global healthcare expenditure on diabetes for 2010 and 2030. Diabetes Res Clin Pract 2010;87:293-301.

11. Islam SMS, Lechner A, Ferrari U, et al. Social and economic impact of diabetics in Bangladesh: protocol for a case-control study. BMC Public Health 2013;13:1217.

12. Shariful Islam SM. Mobile phone interventions for adherence to treatment for diabetics in an urban area of Bangladesh. LMU, 2015. https://edoc.ub.uni-muenchen.de/19148/1/Shariful_Islam_Sheikh_ Mohammed.pdf (accessed 14 Apr 2016).

13. Islam SM, Lechner A, Ferrari U, et al. Mobile phone intervention for increasing adherence to treatment for type 2 diabetes in an urban area of Bangladesh: protocol for a randomized controlled trial. BMC Health Serv Res 2014;14:586.

14. World Health Organization. Process of translation and adaptation of instruments. Secondary Process of translation and adaptation of instruments. 2014. http://www.who.int/substance_abuse/research tools/translation/en/

15. Brown JB, Ramaiya K, Besançon S, et al. Use of medical services and medicines attributable to diabetes in Sub-Saharan Africa. PLOS ONE 2014;9:e106716.

16. Weichle T, Hynes DM, Durazo-Arvizu R, et al. Impact of alternative approaches to assess outlying and influential observations on health care costs. Springerplus 2013;2:1-11.

17. Loganathan A, John K. Economic burden of diabetes in people living with the disease; a field study. J Diabetol 2013; 3:1-8.

18. Brown JB, Nichols GA, Glauber HS, et al. Type 2 diabetes: incremental medical care costs during the first 8 years after diagnosis. Diabetes Care 1999;22:1116-24.

19. American Diabetes Association. Economic costs of diabetes in the US in 2012. Diabetes Care 2013;36:1033-46.

20. Köster I, Von Ferber L, Ihle P, et al. The cost burden of diabetes mellitus: the evidence from Germany-the CoDiM study. Diabetologia 2006;49:1498-504.

21. Misra A, Khurana L. Obesity-related non-communicable diseases: South Asians vs white Caucasians. Int J Obes (Lond) 2010;35:167-87.

22. Mørkrid K, Ali L, Hussain A. Risk factors and prevalence of diabetic peripheral neuropathy: a study of type 2 diabetic outpatients in Bangladesh. Int J Diabetes Dev Ctries 2010;30:11-17.

23. Shariful Islam S, Niessen LW, Seissler J, et al. Diabetes knowledge and glycemic control among patients with type 2 diabetes in Bangladesh. Springerplus 2015;4:284.

24. Werner WJ. Micro-insurance in Bangladesh: risk protection for the poor? J Health Popul Nutr 2009;27:563.

25. van Doorslaer E, O'Donnell O, Rannan-Eliya RP, et al. Effect of payments for health care on poverty estimates in 11 countries in Asia: an analysis of household survey data. Lancet 2006;368:1357-64.

26. Hu JP, Rao KQ, Qian JC, et al. The study of economic burden of chronic non-communicable diseases in China. Chin J Prev Control Chron Non-commun Dis 2007;15:189-93.

27. Zhang Z, Chen J, Tang Z, et al. Study on direct health expenditure of diabetes mellitus in China. Chin Health Res 2007:10:162-8.

28. Mbanya JCN, Motala AA, Sobngwi E, et al. Diabetes in sub-Saharan Africa. Lancet 2010;375:2254-66.

29. Inzucchi S, Bergenstal R, Buse J, et al. Management of hyperglycaemia in type 2 diabetes: a patient-centered approach. Position statement of the American Diabetes Association (ADA) and the European Association for the Study of Diabetes (EASD). Diabetologia 2012;55:1577-96.

30. IDF Clinical Guidelines Task Force. Global guideline for type 2 diabetes. In: Federation ID, ed. Brussels, Belgium: International Diabetes Federation, 2012:123.

31. Islam SMS LA, Ferrari U, Seissler J, et al. Mobile phone use and willingness to pay for SMS for diabetes in Bangladesh. J Public Health (Oxf) 2016;38:163-9.

32. Islam SMS, Niessen LW, Ferrari U, et al. Effects of mobile phone SMS to improve glycemic control among patients with type 2 diabetes in Bangladesh: a prospective, parallel-group, randomized controlled trial. Diabetes Care 2015;38:e112-13.

33. Islam SMS, Tabassum R. Implementation of information and communication technologies for health in Bangladesh. Bull World Health Organ 2015;93:806-9.

34. Rahim M, Hussain A, Azad Khan A, et al. Rising prevalence of type 2 diabetes in rural Bangladesh: a population based study. Diabetes Res Clin Pract 2007;77:300-5. 